\title{
Ist die begleitende Antibiotikagabe bei operativ versorgten Handinfektionen erforderlich?
}

\author{
Manuela Micheel, Theodora Manoli, Hans-Eberhard Schaller
}

\section{Zusammenfassung}

Handinfektionen sind sehr häufige handchirurgische Krankheitsbilder. Anhand der Schwere des Infekts können einfache Handinfektionen ohne Sehnen-, Knochen- oder Gelenkbeteiligung von komplizierten Handinfektionen (septische Tenosynovialitis, septische Arthritis oder Osteomyelitis) unterschieden werden. Grundsätzlich ist das radikale chirurgische Débridement die Therapie der Wahl. Bei unkomplizierten Handinfektionen ist eine begleitende Antibiotikagabe grundsätzlich nicht erforderlich. Liegt eine septische Tenosynovialitis, septische Arthritis oder Osteomyelitis vor bzw. kann der Infekt intraoperativ zum gesunden Gewebe nicht klar abgegrenzt werden, sollte nach erfolgtem chirurgischen Débridement eine systemische und ggf. lokale Antibiotikatherapie erfolgen. Als lokale Antibiotikatherapie hat es sich als effizient erwiesen, drainierende PMMA-Miniketten in die Wunde einzulegen.
Is a Concomitant Antibiotic Therapy after Surgical Treatment of Hand Infections Necessary?

Hand infections are frequent routine hand surgical cases. To simplify the indication for antibiotic administration, hand infections may be classified into uncomplicated infections without affection of tendons, joints or bones and complicated infections including tenosynovialitis, osteomyelitis or septic arthritis. The early radical surgical debridement is the standard treatment for any type of infection. In uncomplicated infections a concomitant administration of antibiotics is usually not necessary. In complicated infections or cases in which the infected tissue could not be debrided sufficiently, antibiotics should be administrated postoperatively. Antibiotics may be applied locally in the form of draining PMMA mini-chains and systemically.

\section{Einleitung}

Die Behandlung von Handinfektionen ist Alltag für Handchirurgen - umso wichtiger ist die rechtzeitige und v.a. richtige Einschätzung der Schwere und einzuleitenden Therapie [1]. Bisher gibt es jedoch keine einheitliche Vorgehensweise oder leitliniengerechte Handhabung bez. der Notwendigkeit einer begleitenden Antibiotikagabe bei operativ versorgten Handinfektionen - dies führt immer wieder zu kontroversen Diskussionen, auch mit anderen Fachdisziplinen [2].

OP-JOURNAL 2015; 31: 99-103

(c) Georg Thieme Verlag KG Stuttgart · New York DOI http://dx.doi.org/10.1055/s-0035-1546004

ihrer Wirksamkeit bei schon vorliegender Infektsituation [6]. Jedoch ist eine frühe adäquate Behandlung einer Handverletzung effektiver als eine begleitende Antibiotikagabe [7]. Vor allem in den USA wird ein zunehmendes Auftreten von MRSA und Keimresistenzen auch bei Handinfektionen berichtet und diskutiert $[8,9]$. Auch aus diesem Grund ist eine standardmäßige Antibiotikagabe zu hinterfragen.

\section{Indikation zur Operation und sachgerechtes operatives Vorgehen}

Gestörte Nachtruhe durch einen lokalisierten pochenden Schmerz an den Fingern oder der Hand, eine fortgeleitete Schmerzsymptomatik entlang der Sehnenscheiden bei der klinischen Untersuchung sowie Überstreckschmerz, d.h. maximale Schmerzsymptomatik bei Überstreckung und Schonhaltung des/ der betroffenen Finger in Beugung sind Indikationen für ein rasches, sachgerechtes chirurgisches Débridement [3]. Des Weiteren sollten sämtliche die Haut bis ins Subkutangewebe perforierende Bissverletzungen, deren Ausdehnung in die Tiefe somit nicht sicher beurteilbar ist (Abb.3), operativ saniert werden. Auch sollten Hochdruckinjektionsverletzungen, mit oder ohne sichtbare Eintrittstelle, notfallmäßig saniert und die zugrunde liegende Substanz ermittelt werden, um ggf. toxikologische Gegenmaßnahmen parallel zum Débridement vornehmen zu können. Das chirurgische Débridement wird grundsätzlich mit Lupenbrille durchgeführt. Im Mittelpunkt steht die Sanierung des Infekts bis über dessen Grenzen ins gesunde Gewebe ohne Rücksicht auf Verlust sämtlicher Strukturen. Die Durchführung eines intraoperativen Abstrichs muss obligat erfolgen. Bei lokal begrenzten Infektionen wird das erkrankte Gewebe radikal bis ins gut durchblutete, gesunde Gewebe entfernt; sämtliche benachbarten Strukturen werden dargestellt und so auf Unversehrtheit überprüft (Sehnen, Nerven, Gefäße und Gelenke; Abb. 1 b). Die Wunde sollte 

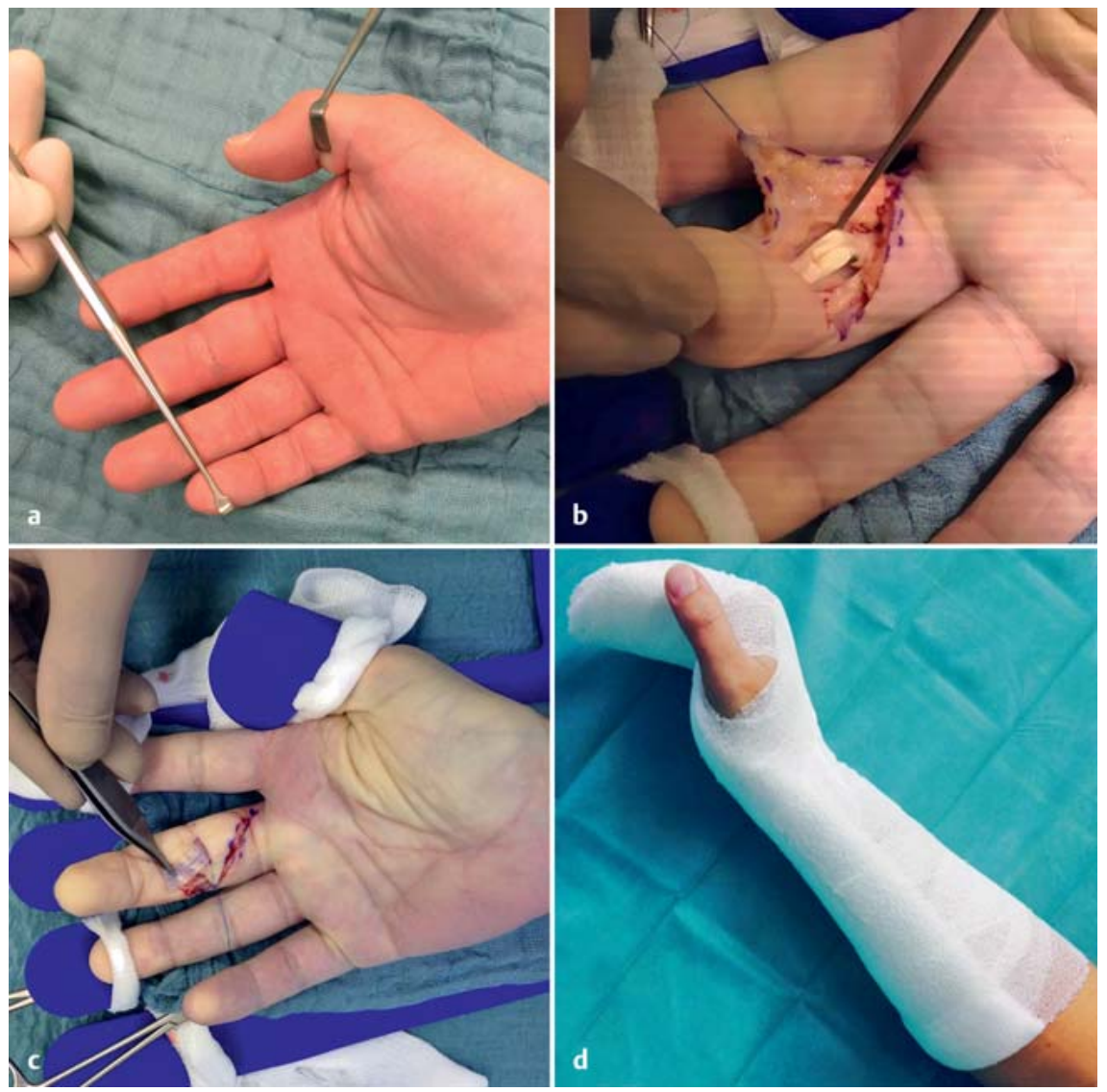

Abb. 1 a bis d a Bagatellverletzung am Mittelfingermittelgelenk rechts palmar. b Nach Exzision der Primärwunde Eröffnung nach Brunner, Darstellung der Beugesehnen und Gefäß-Nerven-Bündel. c Einlage einer Easy-flow-Drainage durch die exzidierte Komplikationswunde am Mittelgelenk. d Ruhigstellung auf einer palmaren 4-Finger-Schiene in Intrinsic-Stellung.

ausgiebig auf mögliche Fremdkörper inspiziert werden. Die Schnittführung am Finger erfolgt nach Bruner (Abb. 1 c) oder mediolateral und kann bei Ausweitung des Infekts bis in die Hohlhand fortgesetzt werden (Abb. 2). Liegt eine Teno- synovialitis vor (Abb. 2 und Abb.4a), sollte die betroffene Sehnenscheide mittels eines Spülkatheters gereinigt werden werden, dies sollte mehrmals und von proximal nach distal erfolgen. Es können nachfolgend gentamycinbe- schichtete PMMA-Miniketten zur Sekretableitung in die Wunde eingelegt werden (Abb. 4 b). Zeigt sich die Sehne intraoperativ lytisch (Abb.5b), muss auch diese entfernt und in weiteren Eingriffen nach Infektsanierung ggf. rekonstruiert werden. Auch bei Vorhandensein einer Arthritis bzw. Osteomyelitis werden die betroffenen Knochenteile radikal entfernt, hiernach sollte eine temporäre Arthrodese mittels eines Fixateur externe (Abb.6a und b) angestrebt werden, ggf. ist auch eine Ruhigstellung des betroffenen Gelenks zur Einsteifung in Funktionsstellung sinnvoll. Bleibt nach $\mathrm{Ab}$ klingen des Infekts eine zeitgerechte knöcherne Konsolidierung aus, sollte die Durchführung einer funktionsgerechten Arthrodese geplant werden [10]. Die Radikalität eines Débridements bei Infektionen der Hand sollte wie bei onkologischen Operationen erfolgen, da ein $\mathrm{Zu}$ rücklassen von infiziertem Gewebe zu einer unkontrollierten Ausbreitung des Infekts führen kann. Bei chirurgisch nicht sicher beherrschbarer Wunde bzw. nicht eindeutig bestimmbaren Infektgrenzen (Abb. 5 b und 7) sollte großzügig die Indikation zum Second bzw. Third Look gestellt werden [3]. Die Wundränder sollten beim Wundverschluss locker adaptiert und die Nähte nicht zu eng gesetzt werden, ggf. ist bei größeren HautWeichteil-Defekten später eine plastisch-chirurgische Deckung erforderlich.

Je nach Ausbreitung der Infektion, Infiltration der verschiedenen Strukturen bzw. Beherrschbarkeit der vorliegenden Infektsituation sollte die Indikation $\mathrm{zu}$ einer das chirurgische Débridement be-

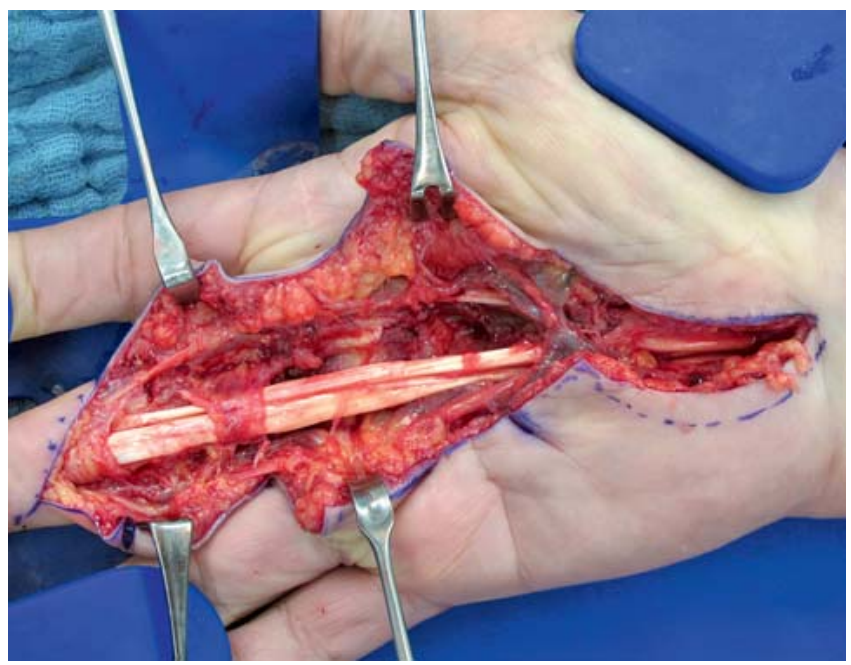

Abb. 2 Tenosynovialitis nach ausgiebigem chirurgischem Débridement und Spülung mittels Spülkatheter bei ausgeprägtem Befund. Eröffnung am Finger nach Bruner mit Schnitterweiterung bis in die Hohlhand und Karpaltunnelspaltung.

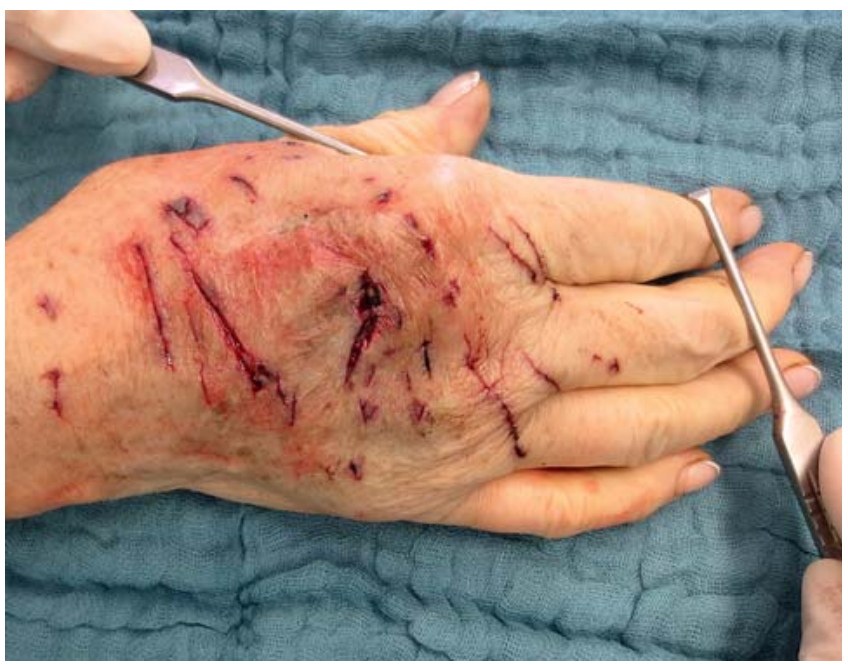

Abb. 3 Multiple tiefreichende Katzenkratz- und -bissverletzungen Handrücken rechts vor Exzision der Bisskanäle und Einlage der PMMAMiniketten. 
gleitenden Antibiotikatherapie erfolgen. Im Folgenden möchten wir deshalb Handinfektionen nach ihrem Schweregrad klassifizieren und die jeweilige empfohlene antibiotische Therapie nach chirurgischer Sanierung der Handinfektion diskutieren.

\section{Klassifikation von Handinfektionen}

Handinfektionen werden in der Literatur meist nach ihrer Lokalisation oder Art der Verletzung klassifiziert, z. B. das Panaritium, die Tenosynovialitis, die Hohlhandphlegmone oder die Bissverletzung [7]. Manoli et al. beschrieben 2013 ein globales Klassifikationssystem, das die Schwere eines Handinfekts je nach Ausbreitung im Gewebe bestimmt [5]. Anhand dieser Klasseneinteilung empfahlen die Autoren das einheitliche Vorgehen hinsichtlich einer Antibiotikagabe bei Handinfektionen (Tab. 1).

\section{Schweregradgerechte antibiotische Behandlung}

\section{Unkomplizierte Handinfektionen}

Bei unkomplizierten Handinfektionen ohne das Vorhandensein von Tenosynovialitis, Arthritis oder Osteomyelitis hat sich eine intra- bzw. postoperative Gabe von Antibiotika bei sachgerecht durchgeführtem operativen Débridement grundsätzlich als nicht notwendig erwiesen [5]. Vielmehr können in diesen Fällen bei Bedarf Drainagen zur Sekretableitung in die Wunden gelegt werden, z. B. Easy-flow-Drainagen (Abb. 1 c).

Im Rahmen einer prospektiv durchgeführten Patientendatenerfassung konnte gezeigt werden, dass in Bezug auf Revisionsrate, Ergebnis des DASH-Scores und Rekonvaleszenz keine Vorteile der mit lokal (gentamycinbeschichteten PMMA-Miniketten, Abb. 4b) oder systemisch antibiotisch behandelten Kontrollgruppen gegenüber der Gruppe ohne Antibiotikagabe bestanden [5]. So kann auch die Bildung von Antibiotikaresistenzen vermindert werden, was insbesondere mit dem vermehrten Auftreten von methicillinresistenten Staphylococcus aureus (MRSA) zunehmend an Bedeutung gewinnt $[8,9,11]$.

Ausnahmen stellen Patienten dar, bei denen immunschwächende (Immunsuppressiva, Polytoxikomanie) oder wundheilungsstörende Faktoren Abb.7, wie z.B. ein schlecht eingestellter Diabetes mellitus, vorliegen (Abb. 5a und b) [12].

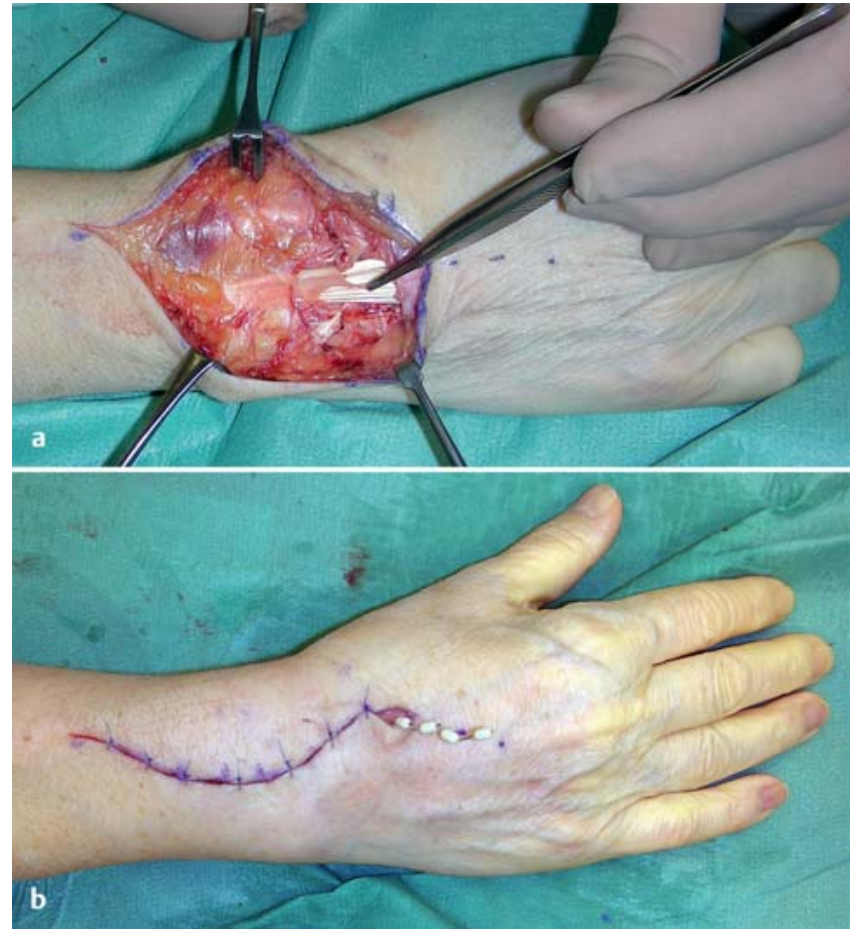

Abb. $4 a$ und $b$ a Tenosynovialitis der Strecksehnen während des chirurgischen Débridements. b Wundverschluss mittels locker adaptierender Nähte und Einlage einer PMMA-Minikette.

Hier sollte die Entscheidung zur begleitenden Antibiotikagabe großzügiger gestellt werden.

\section{Komplizierte Handinfektionen mit Tenosynovialitis und/oder Osteomyelitis}

Für die komplizierten Handinfektionen mit Tenosynovialitis (Klasse II) und/oder Osteomyelitis (Klasse III) sowie bei Infekten, die intraoperativ nicht eindeutig vom gesunden Gewebe abzugrenzen sind, sollte nach operativem Débridement und Abstrichentnahme eine begleitende systemische und ggf. lokale Antibiotikagabe erfolgen $[5,10]$. So kann verhindert werden, dass es bei Hinterlassen von infiziertem Gewebe durch unzureichendes Débridement zu einer unkontrollierten Ausbreitung des Infekts kommt [10].

Tab. 1 Einteilung der Handinfektionen nach Schweregrad und darauf abgestimmte empfohlene chirurgische bzw. antibiotische Therapie nach Manoli et al. 2013.

\begin{tabular}{llll} 
Klasse & Merkmale & operatives Vorgehen & Antibiose \\
\hline I & $\begin{array}{l}\text { kutan, subkutan oder subfaszial be- } \\
\text { grenzte Handinfektionen ohne Teno- } \\
\text { synovialitis, Arthritis oder Osteomyelitis }\end{array}$ & $\begin{array}{l}\text { radikales Débridement, } \\
\text { ggf. Drainageneinlage }\end{array}$ & nein \\
\hline II & $\begin{array}{l}\text { Tenosynovialitis ohne Arthritis oder } \\
\text { Osteomyelitis, fakultativ Symptome } \\
\text { wie bei Klasse I }\end{array}$ & $\begin{array}{l}\text { radikales Débridement, } \\
\text { ggf. Einlage eines Spül- } \\
\text { katheters }\end{array}$ & ja \\
\hline III & $\begin{array}{l}\text { septische Arthritis oder Osteomyelitis, } \\
\text { fakultativ Symptome wie in Klasse I } \\
\text { und/oder Klasse II }\end{array}$ & $\begin{array}{l}\text { radikales Débridement, } \\
\text { ggf. septische Arthrodese } \\
\text { oder Stumpfbildung }\end{array}$ & ja \\
& &
\end{tabular}




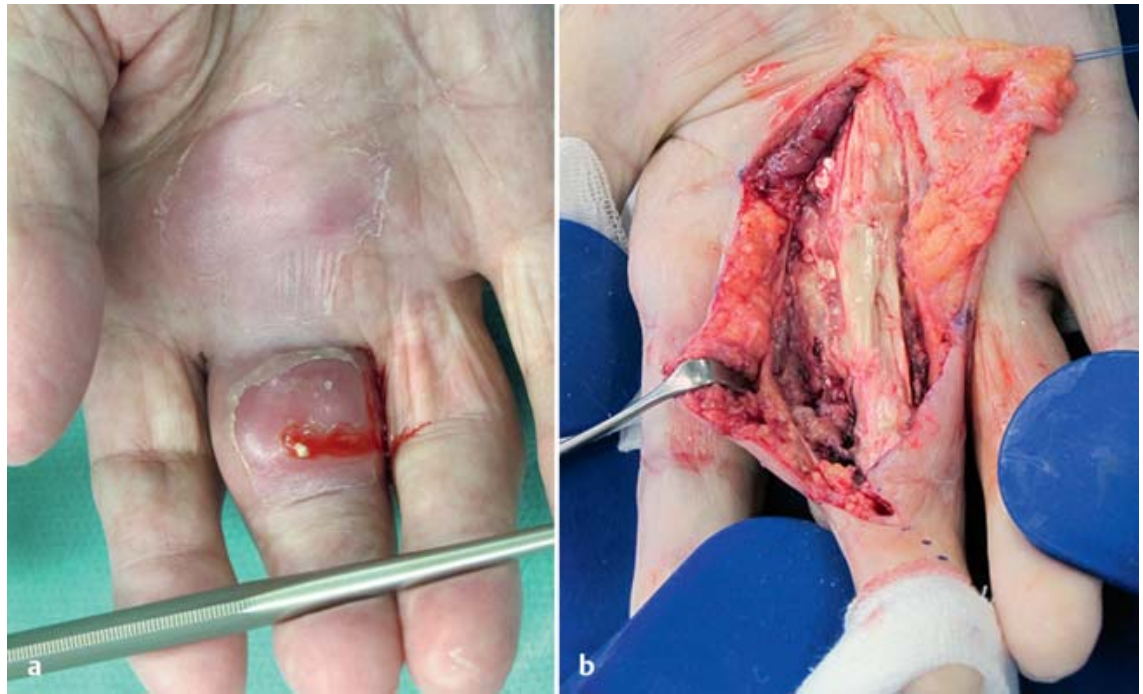

Abb.5a und $\mathbf{b}$ a Bagatellverletzung vor mehreren Tagen bei einem Patienten mit Diabetes mellitus. Schwellung und pochende Schmerzen seit 2 Tagen, seit Vortag Pusaustritt Grundglied Mittelfinger rechts. $\mathbf{b}$ Lytische Beugesehnen, welche radikal debridiert und zur Beherrschung des Infektes entfernt werden müssen. Nach Abklingen der Infektsituation ist die zweizeitige Rekonstruktion mittels Silastik-Stab und Palmaris-Longus-Transplantat notwendig.
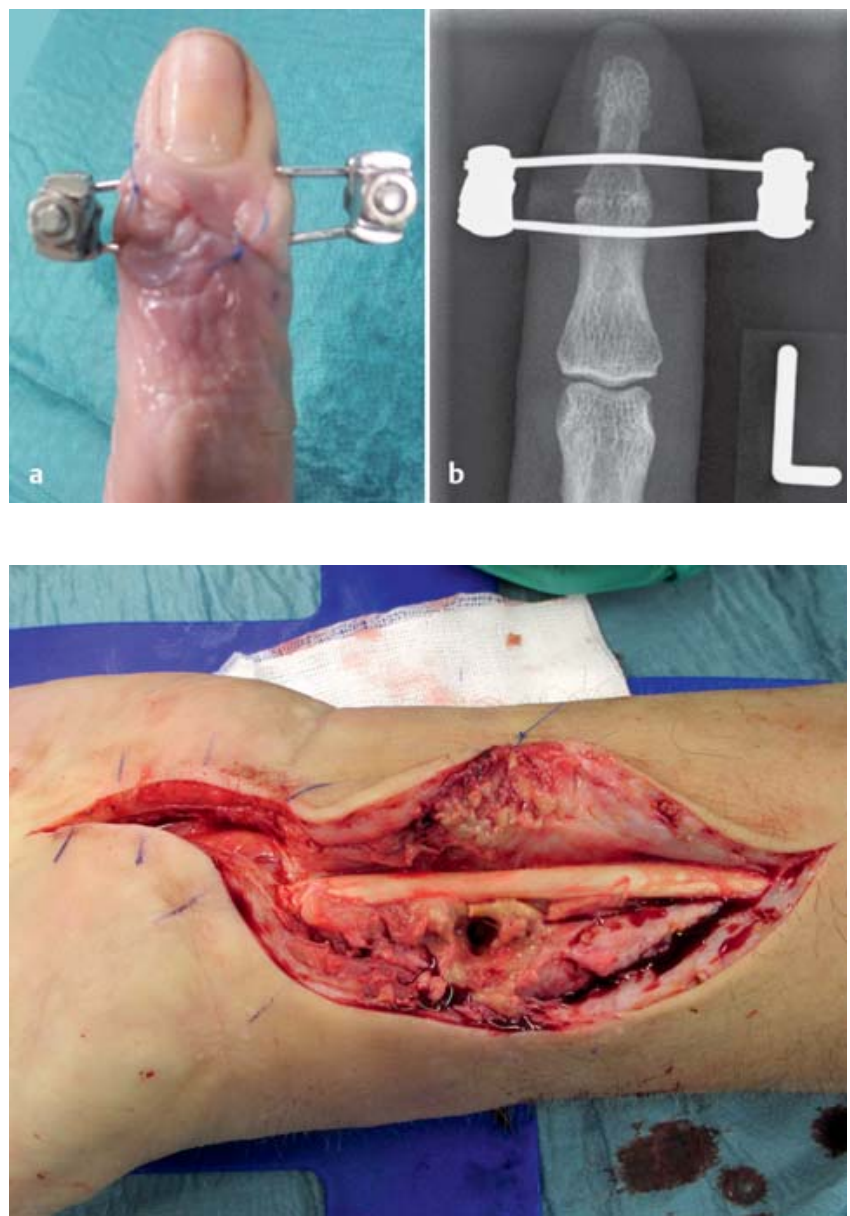

Abb. 7 Handgelenk51-jährigen Patienten eine Nadelstichverlet-

Bei Bissverletzungen wird das Erregerspektrum durch Kombinationspräparate eines Aminopenicillins mit einem $\beta$ Lactamase-Inhibitor meist besser abgedeckt, da hier Pasteurellen die häufigsempyem bei einem mit Polytoxikomanie vermutlich durch zung mit Pannusgewebe entlang der Beugesehnen und Einschmelzung der

A. radialis.

Abb. 6 a und $b$ Septische Arthrodes gelenks links mittels Mini-Fixateur externe nach Dorninkorporation vor ca. 4 Wochen. jeder Handinfektion präoperativ ein $\mathrm{Ab}$ strich zu nehmen ist, ist nach gesichertem Erregernachweis ein Wechsel der Antibiose angezeigt, falls zugrunde liegende Keime durch das Antibiotikum nicht abgedeckt waren und die Zeichen der Infektion noch nicht abgeklungen sind. Liegt eine ausgeprägte eitrige Tenosynovialitis oder Osteomyelitis vor, wird die intravenöse Antibiose oft länger als 5 Tage, für mindestens 7-14 Tage, verabreicht [1].

\section{Antibiotikatherapie bei konservativ geführten Handinfektionen}

Im Falle von beginnenden, nicht eitrigen und lokal begrenzten Infektionen kann zunächst ein konservativer Therapieversuch unternommen werden. Dies ist nur bei fehlendem Überstreckschmerz, fehlenden fortgeleiteten Entzündungszeichen und fehlendem pochenden Nachtschmerz angezeigt. Das Behandlungskonzept besteht aus der konsequenten Ruhigstellung in einer Gipsschiene, körperlicher Schonung, Hochlagerung und Kühlung der betroffenen Extremität. Eine tägliche fachchirurgische Befundkontrolle ist unabdingbar. Bei Persistenz der Beschwerden oder Fortschreiten der Entzündungszeichen ist das chirurgische Débridement unumgänglich [3].

Auf eine Antibiotikagabe sollte bei primär konservativ geführten Handinfektionen jedoch verzichtet werden, da eine alleinige Antibiotikagabe die Ausbreitung der Handinfektion oft nicht effektiv aufhalten, sondern sogar zu deren Verschleierung beitragen kann [12]. Dies liegt darin begründet, dass Antibiotika in den oberflächlichen Schichten, die besser perfundiert sind als tiefere Strukturen wie Faszien, Sehnen und Knochen, eine höhere Wirkung aufweisen. Somit wird bei deren Gabe unter Umständen eine Ausbreitung des Infekts begünstigt, ohne dass sich die äußerlichen Infektzeichen verschlechtert haben. Die Indikationsstellung zum operativen Débridement wird dann u.U. zu spät gestellt [3].

Immunsupprimierte Patienten bzw. Patienten mit Krankheiten, welche die Wundheilung negativ beeinflussen (z.B. ein schlecht eingestellter Diabetes mellitus) stellen hinsichtlich eines konservativen Therapieversuchs wiederum eine Ausnahme dar. Hier sollte die Indikation zum primären operativen Débridement und postoperativen Antibiotikagabe großzügiger gestellt werden. 


\section{Nachbehandlung}

Bei sämtlichen Handinfektionen ist eine strenge und konsequente Nachbehandlung ebenso wichtig wie die operative Sanierung des Infekts. Postoperativ sollte Bettruhe eingehalten sowie die betroffene Extremität bis zum Abklingen der lokalen Entzündungszeichen in einer angepassten Unterarmschiene mit Einschluss sämtlicher Finger bzw. des Daumens in Intrinsic-Plus-Stellung ruhiggestellt werden.

Kommt es unter Einhaltung des beschriebenen postoperativen Therapiekonzepts zur Progredienz oder besteht eine Persistenz der Entzündungszeichen, sollte großzügig die Indikation zum Second Look erfolgen [3]. In diesem Fall ist dann eine nach Antibiogramm bestimmte postoperative Antibiotikagabe sinnvoll.

\section{Schlussfolgerung}

Bei unkomplizierten Handinfektionen ohne Sehnen-, Knochen- oder Gelenkbeteiligung ist eine begleitende Antibiotikagabe nach chirurgischem Débridement nicht zwingend nötig. Liegt eine eitrige Tenosynovialitis, Arhtritis oder Osteomyelitis vor bzw. kann der Infekt intraoperativ zum gesunden Gewebe nicht klar abgegrenzt werden, sollte nach Abstrichentnahme und radikalem operativem Débridement eine begleitende systemische Antibiotikagabe erfolgen. In diesen Fällen hat es sich als effizient bewiesen, drainierende PMMA-Miniketten lokal in die Wunde einzulegen.

\section{Literatur}

${ }^{1}$ McDonald LS, Bavaro MF, Hofmeister EP et al. Hand infections. J Hand Surg Am 2011; 36: 1403-1412

2 Paydar KZ, Hansen SL, Charlebois ED et al. Inappropriate antibiotic use in soft tissue infections. Arch Surg 2006; 141: 850-854

3 Schaller H-E. Infekte der Hand. Trauma Berufskrankh 2008; 10: 146-150

${ }^{4}$ Kennedy SA, Stoll LE, Lauder AS. Human and other mammalian bite injuries of the hand: evaluation and management. J Am Acad Orthop Surg 2015; 23: 47-57

5 Manoli T, Rahmanian-Schwarz A, Konheiser K et al. The role of antibiotics after surgical treatment of simple hand infections: a prospective pilot study. J Invest Surg 2013; 26: 229-234

6 Platt AJ, Page RE. Post-operative infection following hand surgery. Guidelines for antibiotic use. J Hand Surg Br 1995; 20: 685-690

7 Rigopoulos N, Dailiana ZH, Varitimidis S et al. Compartmental infections of the hand. Scand J Plast Reconstr Surg Hand Surg 2008; 42: 38-42

8 Janis JE, Hatef DA, Reece EM et al. Does empiric antibiotic therapy change MRSA hand infection outcomes? Cost analysis of a random- ized prospective trial in a county hospital. Plast Reconstr Surg 2014; 133: 511e-518e

${ }^{9}$ Harrison B, Ben-Amotz O, Sammer DM. Methicillin-resistant staphylococcus aureus infection in the hand. Plast Reconstr Surg 2015; 135: 826-830

10 Manoli T, Schaller H-E. Allgemeine Handinfektionen. In: Sauerbier M, Eisenschenk A, Krimmer H, Partecke B-D, Schaller H-E. Die Handchirurgie. München: Elsevier; 2014: 172-192

${ }^{11}$ Rigopoulos N, Dailiana ZH, Varitimidis S et al. Closed-space hand infections: diagnostic and treatment considerations. Orthop Rev 2012; 4: e19

12 Stahl S, Held M, Medved F et al. Infektionen nach sogenannten Bagatellverletzungen der Hand. Trauma Berufskrankh 2014; 16: 281286

\section{Dr. med. Manuela Micheel}

Assistenzärztin

Dr. med. Theodora Manoli

Oberärztin

Prof. Dr. med. Hans-Eberhard

Schaller

Direktor der Klinik

Klinik für Hand-, Plastische, Rekonstruktive und Verbrennungschirurgie Eberhard-Karls-Universität Tübingen/

BG Unfallklinik

Schnarrenbergstraße 95

72076 Tübingen

mmicheel@bgu-tuebingen.de 